This item was submitted to Loughborough's Research Repository by the author.

Items in Figshare are protected by copyright, with all rights reserved, unless otherwise indicated.

\title{
Modelling of instabilities in turbulent swirling flames
}

PLEASE CITE THE PUBLISHED VERSION

PUBLISHER

(C) Elsevier

VERSION

AM (Accepted Manuscript)

LICENCE

CC BY-NC-ND 4.0

REPOSITORY RECORD

Ranga-Dinesh, K.K.J., K.W. Jenkins, M.P. Kirkpatrick, and W. Malalasekera. 2019. "Modelling of Instabilities in Turbulent Swirling Flames". figshare. https://hdl.handle.net/2134/5496. 
This item was submitted to Loughborough's Institutional Repository (https://dspace.lboro.ac.uk/) by the author and is made available under the following Creative Commons Licence conditions.

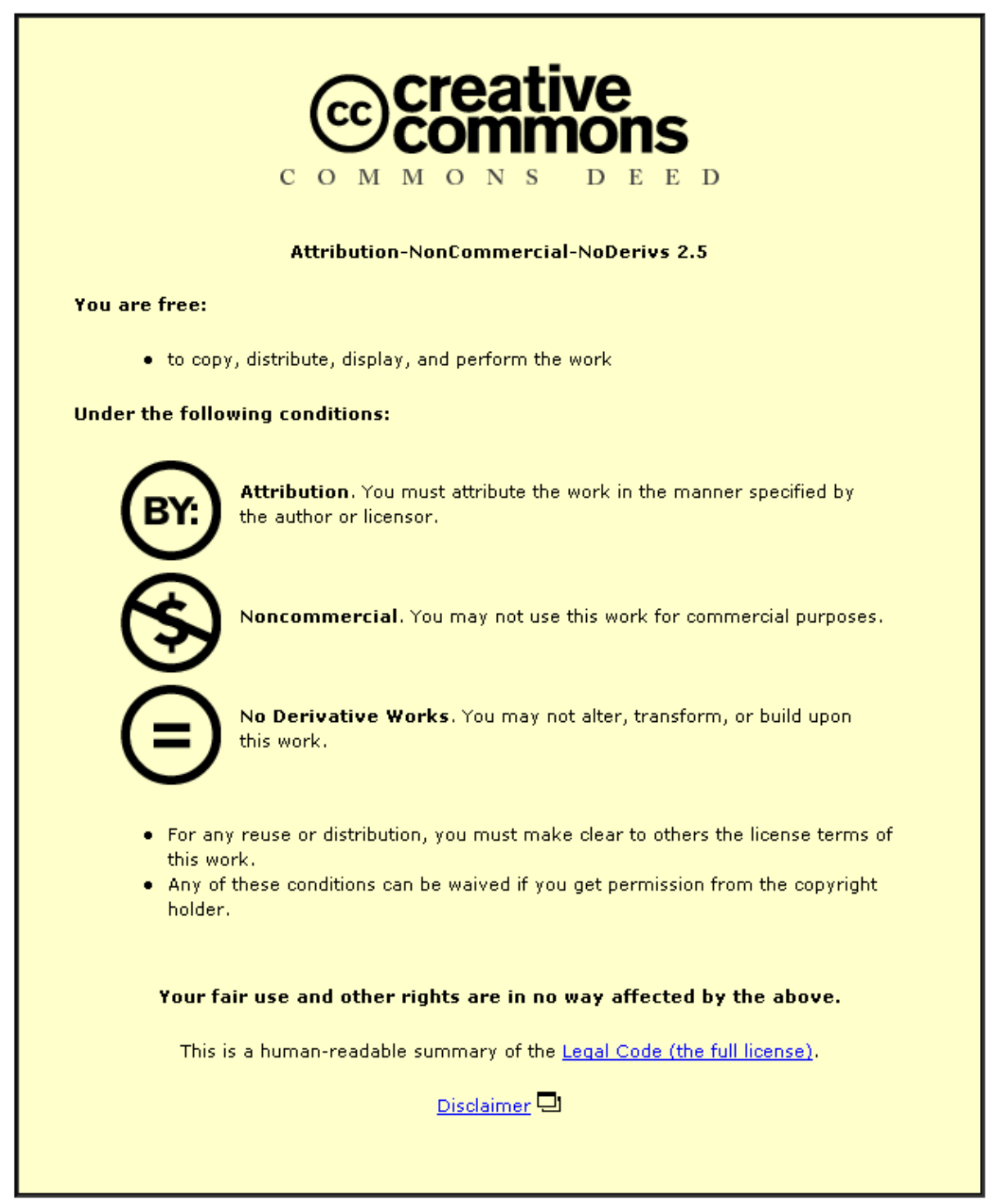

For the full text of this licence, please go to: http://creativecommons.org/licenses/by-nc-nd/2.5/ 


\section{Modelling of Instabilities in Turbulent Swirling Flames}

K.K.J.Ranga Dinesh ${ }^{1}$, K.W.Jenkins ${ }^{1}$, M.P.Kirkpatrick ${ }^{2}$, W.Malalasekera ${ }^{3}$

1. School Of Engineering, Cranfield University, Cranfield, Bedford, MK43 0AL, UK

2. School of Aerospace, Mechanical and Mechatronic Engineering, The University of Sydney, NSW 2006, Australia

3. Wolfson School of Mechanical and Manufacturing Engineering, Loughborough University, Loughborough, Leicester, LE11 3TU, UK

Corresponding author: K.K.J.Ranga Dinesh

Email address: Ranga.Dinesh@Cranfield.ac.uk

Postal Address: School of Engineering, Cranfield University, Cranfield, Bedford, MK43 0AL, UK.

Telephone number: +44 (0) 1234750111 ext 5350

Fax number: +44 1234750195

Revised Manuscript prepared for the Journal of Fuel

$5^{\text {th }}$ May 2009 


\title{
Modelling of Instabilities in Turbulent Swirling Flames
}

\author{
K.K.J.Ranga Dinesh ${ }^{1}$, K.W.Jenkins ${ }^{1}$, M.P.Kirkpatrick ${ }^{2}$, W.Malalasekera ${ }^{3}$
}

\begin{abstract}
A large eddy simulation based data analysis procedure is used to explore the instabilities in turbulent non-premixed swirling flames. The selected flames known as SM flames are based on the Sydney swirl burner experimental database. The governing equations for continuity, momentum and mixture fraction are solved on a structured Cartesian grid and the Smagorinsky eddy viscosity model with dynamic procedure is used as the subgrid scale turbulence model. The thermo-chemical variables are described using the steady laminar flamelet model. The results show that the LES successfully predicts the upstream first recirculation zone generated by the bluff body and the downstream second recirculation zone induced by swirl. Overall, LES comparisons with measurements are in good agreement. Generated power spectra and snapshots demonstrate oscillations of the centre jet and the recirculation zone. Snapshots of flame SM1 showed irregular precession of the centre jet and the power spectrum at a downstream axial location situated between the two recirculation zones showed distinct precession frequency. Mode II instability defined as cyclic expansion and collapse of the recirculation zone is also identified for the flame SM2. The coupling of swirl, chemical reactions and heat release exhibits Mode II instability. The presented simulations demonstrate the efficiency and applicability of the LES technique to swirl flames.
\end{abstract}

Key words: Swirl, Instability, Precession, Combustion, Large eddy simulation 


\section{Introduction}

The occurrence of oscillations and instabilities in a swirl combustion system presents a technical challenge for the development of engineering applications such as gas turbines, IC engines, furnaces etc. Strong interactions occur between the flow field and flame which increases the complexity of combustion oscillations and may even lead to failure in some situations such as those encountered in low-emission, leanpremixed systems [1]. The occurrence of recirculation and vortex breakdown (VB) rotates the heat release distribution and active chemical species to the root of the flame and produces a relatively compact flame. The presence of the precessing vortex core (PVC), in which the centre of the vortex precesses around the centre axis of symmetry, further increases the instability in swirl combustion systems.

Although the nature of instabilities in both reacting and non-reacting swirling flow fields have been studied during the past few decades, further investigations for important issues such as jet precession, PVC, instability modes and vortex-flame interactions are needed [2]. Theoretical and experimental studies have been carried out to discover the types of instabilities for reacting and non-reacting swirling flows and they found that the influence of swirl depends on different flow parameters such as inflow velocity profiles, Reynolds number, level of swirl and geometrical configuration. A number of papers exist on these topics ranging from VB to instabilities of swirling flows [2-4].

Computational fluid dynamics (CFD) is now widely used in combustion applications and the rise in large scale computing resources has permitted the simulations of flows encountered in realistic swirl stabilised combustion systems [5-8]. Also, advanced state-of-art numerical techniques with sophisticated algorithms provide a more 
efficient and effective design tool for swirl based combustion systems, which reduces the larger cost associated with expensive experimental testing. Large eddy simulation (LES) is a promising tool for studying the underlying physics of unsteady flow problems. LES is often considered to be the natural successor to methods based on Reynolds Navier-Stokes (RANS) as it can potentially address the inherent unsteadiness of physical properties in a turbulent flow with sufficient spatial and temporal accuracy for combustion applications [9-10]. In LES, the large scale turbulence structures are directly computed and small dissipative structures are modelled. With rapid development of computer hardware, LES is more applicable for high Reynolds number complex engineering problems than expensive direct numerical simulation (DNS) in which all scales are resolved with high accuracy. LES is also more accurate than conventional Reynolds averaged Navier-Stokes (RANS) in which only the mean quantities are computed.

During the past ten years LES has been applied to a variety of swirling applications in both the reacting and non-reacting situations. For non-reacting swirl applications, Lu et al. [11] have shown encouraging results for different swirl conditions in a confined swirl combustor configuration and found useful details for the shear layer instability and vortex-acoustic interactions. Wang et al. [12] carried out a comprehensive study on confined swirling flows in an operational gas turbine combustor. Encouraging results have been reported in recent literature [13-16], which demonstrate the ability of LES to capture the unsteady swirling flame structure in a complex swirl configuration including multiphase flows and combustion processes such as gas turbine combustion, internal combustion engines, industrial furnaces and liquid-fueled rocket propulsion. Furthermore, Pierce and Moin [17] used a flamelet model 
combined with a progress variable and obtained encouraging results for gaseous swirling flames. Sankaran and Menon [5] carried out LES for a non-premixed swirling spray combustion and Oefelein et al. [7], Oefelein [13] also developed a unique technique for the application of LES on spray combustion. Apte et al. [6] carried out LES calculations for swirling particle laden flow in a co-axial combustor and Reveillon and Vervisch [18] presented spray vaporisation in non-premixed flames with a single droplet model. Angelberger et al. [19] studied the combustion instabilities using LES technique for premixed flames.

The primary objective of this work is to identify the instabilities experienced in a laboratory scale swirl burner and demonstrate the predictive capability of LES to capture centre jet precession, recirculation zone precession and VB of swirling flames. The laboratory burner used in this study is the Sydney swirl burner, which is frequently used in the study of swirling, combusting flows [20-22]. The swirl burner data base provides a perfect platform for the computational researchers to validate their numerical tools for the highly unsteady reacting and non-reacting complex swirling flow fields. A number of LES studies have attempted to validate the calculations from the Sydney burner experimental data base. For example, El-Asrag and Menon [23] simulated a few flames using the linear eddy combustion model, James et al. [24] modelled selected swirling flames using the filtered density function approach. In earlier studies [25] , presented a comprehensive data analysis for the time-averaged mean velocities and rms fluctuations for the isothermal swirling jets, which was later extended to LES of reacting flames and good agreement was achieved with the experimental data [26]. In addition, flame comparisons from two independent LES codes, which employed different turbulence inflow generation 
methods, different numerical schemes and different grid resolutions have been studied and both groups found good agreement with the experimental data and captured the flow properties such as recirculation and VB including basic flame structure [27]. Although the flow structures and flame properties for the Sydney swirl data base have been computationally investigated, a comprehensive data analysis is required to identify the instabilities that occur for these flames. Ranga Dinesh and Kirkpatrick [28] recently examined the instability of isothermal swirling jets using LES and captured precession motion of central jet and identified PVC structures for a range of swirl numbers. As reported in literature [1][2][3], the addition of combustion for the swirling jet is known to increase the complexity of dynamics with the occurrence of heat release and thus gives rise to varies instability mechanisms. With this in mind, the present work continues our previous work [28] and investigates the instability associated with swirling flames using LES methodology. The selected flame series known as pure methane (SM) which contains two different flames known as SM1 and SM2 [20][22]. In this paper, the instability of the central fuel jet and bluff body stabilised recirculation zone will be addressed using the time varying (unsteady) behaviour of the central jet and time varying (unsteady) behaviour of the bluff body stabilised recirculation zone.

In the following sections, the experimental details of the Sydney swirl burner, theoretical formulations and numerical details are described. Results from the LES calculations are discussed and finally the key conclusions of this study are summarised. 


\section{The Sydney swirl burner}

The Sydney swirl burner configuration shown in Fig. 1, is an extension of the wellcharacterized Sydney bluff body burner to the swirling flames. Extensive details have been reported in the literature and will only be summarised here [20-22]. The burner has a $60 \mathrm{~mm}$ diameter annulus for a primary swirling air stream surrounding the circular bluff body of a diameter $\mathrm{D}=50 \mathrm{~mm}$. The central fuel jet is $3.6 \mathrm{~mm}$ in diameter. The burner is housed in a secondary co-flow wind tunnel with a square cross section with $130 \mathrm{~mm}$ sides. Swirl is introduced aerodynamically into the primary annulus air stream at a distance 300mm upstream of the burner exit plane and inclined 15 degrees upward to the horizontal plane. The swirl number can be varied by changing the relative magnitude of the tangential and axial flow rates. In the present LES calculations, the SM flame series was modelled. Methane is considered to be the fuel in these flames and the properties of the simulated flames are summarised in table 1. Where, $U_{j}(m / s), U_{s}(m / s), W_{s}(m / s), U_{e}(m / s), S_{g}$, and Re are fuel jet velocity, axial velocity of the primary annulus, swirl velocity of the primary annulus, secondary co-flow velocity, swirl number and Reynolds number of the fuel jet respectively. Here the swirl number of the primary annulus is defined as $S_{g}=W_{s} / U_{s}$ and Reynolds number of the jet is defined as $\operatorname{Re}=U_{j} D / v$, where $\mathrm{D}$ is the jet diameter $3.6 \mathrm{~mm}$ and $v$ is the kinematic viscosity.

\begin{tabular}{|l|l|l|l|l|l|l|l|}
\hline Case & Fuel & $U_{j}(m / s)$ & $U_{s}(m / s)$ & $W_{s}(m / s)$ & $U_{e}(m / s)$ & $S_{g}$ & $\operatorname{Re}$ \\
\hline SM1 & $\mathrm{CH}_{4}$ & 32.7 & 38.2 & 19.1 & 20.0 & 0.5 & 7,200 \\
\hline SM2 & $\mathrm{CH}_{4}$ & 88.4 & 38.2 & 19.1 & 20.0 & 0.5 & 19,500 \\
\hline
\end{tabular}

Table 1 Details about the characteristics properties of SM flame series 


\section{Theoretical formulations and numerical details}

For the LES approach, the flow variables are decomposed into the resolved and unresolved (subgrid-scale) components by a spatial filter. The transport equations for Favre filtered mass, momentum and mixture fraction are given by

$$
\begin{gathered}
\frac{\partial \bar{\rho}}{\partial t}+\frac{\partial \bar{\rho} \tilde{u}_{j}}{\partial x_{j}}=0 \\
\frac{\partial \bar{\rho}_{i}}{\partial t}+\frac{\partial\left(\bar{\rho} \tilde{u}_{i} \tilde{u}_{j}\right)}{\partial x_{j}}=-\frac{\partial \bar{P}}{\partial x_{i}}+\frac{\partial}{\partial x_{j}}\left[\overline{2 \rho}\left(v+v_{t}\right)\left[\frac{1}{2}\left(\frac{\partial \tilde{u}_{i}}{\partial x_{j}}+\frac{\partial \tilde{u}_{j}}{\partial x_{i}}\right)-\frac{1}{3} \delta_{i j} \frac{\partial \tilde{u}_{k}}{\partial x_{k}}\right]\right] \\
+\frac{1}{3} \frac{\partial}{\partial x_{j}}\left[\bar{\rho} \delta_{i j} \tau_{k k}\right]+\bar{\rho} g_{i}
\end{gathered}
$$

The transport equation for conserved scalar mixture fraction is written as

$$
\frac{\partial \tilde{\rho} \tilde{f}}{\partial t}+\frac{\partial}{\partial x_{j}}\left(\bar{\rho} \tilde{u}_{j} \tilde{f}\right)=\frac{\partial}{\partial x_{j}}\left[\bar{\rho}\left(\frac{v}{\sigma}+\frac{v_{t}}{\sigma_{t}}\right) \frac{\partial \tilde{f}}{\partial x_{j}}\right]
$$

In the above equations $\rho$ is the density, $u_{i}$ is the velocity component in $x_{i}$ direction, $p$ is the pressure, $v$ is the kinematics viscosity, $f$ is the mixture fraction, $v_{t}$ is the turbulent viscosity, $\sigma$ is the laminar Schmidt number, $\sigma_{t}$ is the turbulent Schmidt number and $\tau_{k k}$ is the isotropic part of the sub-grid scale stress tensor. An over-bar describes the application of the spatial filter while the tilde denotes Favre filtered quantities. The laminar Schmidt number was set to 0.7 and the turbulent Schmidt number for mixture fraction was set to 0.4. The subgrid contribution to the momentum flux is computed using the Smagorinsky eddy viscosity model [29], which uses a model constant $C_{s}$, the filter width $\Delta$ and strain rate tensor $S_{i, j}$ such that

$$
v_{t}=C_{s} \Delta^{2}\left|S_{i, j}\right|=C_{s} \Delta^{2}\left|\frac{1}{2}\left(\frac{\partial \tilde{u}_{i}}{\partial x_{j}}+\frac{\partial \tilde{u}_{j}}{\partial x_{i}}\right)\right|
$$


The model parameter $C_{s}$ is obtained through the localised dynamic procedure of Piomelli and Liu [30].

In LES, the chemical reactions occur mostly in the sub-grid scales and therefore consequent modelling is required for combustion chemistry. Here an assumed probability density function (PDF) of the mixture fraction is chosen as a means of modelling the sub-grid scale mixing. A $\beta$ function is used for the mixture fraction PDF. The functional dependence of the thermo-chemical variables are closed through the steady laminar flamelet approach. In this approach the density, temperature and species concentrations only depend on Favre filtered mixture fraction, mixture fraction variance and scalar dissipation rate. The flamelet calculations were performed using the FlameMaster code [31] incorporating the GRI 2.11 mechanism for detailed chemistry [32]. The sub-grid scale variance of the mixture fraction is modelled assuming the gradient transport equation proposed by Branly and Jones [33].

The LES code called PUFFIN developed by Kirkpatrick [34] and extended by Ranga Dinesh [35] computes the temporal development of the large-scale flow structures by solving the transport equations for the spatially filtered mass, momentum and mixture fraction. A top hat filter with a filter-width equal to the size of the local cell is used. The equations are discretised in space with a finite volume formulation (FVM) using Cartesian coordinates on a non-uniform staggered grid. A second order central difference scheme (CDS) is used for the spatial discretisation of all terms in both the momentum equation and the pressure correction equation. This minimises the projection error and ensures convergence in conjunction with an iterative solver. The diffusion terms of the scalar transport equation are also discretised using a second 
order CDS, and the advection term of the mixture fraction transport equation is discretised using the SHARP scheme [36]. An iterative time advancement scheme is used for the variable density calculation. The time derivative of the mixture fraction is approximated using the Crank-Nicolson scheme. The momentum equations are integrated in time using a second order hybrid scheme. Advection terms are calculated explicitly using a second order Adams-Bashforth while diffusion terms are calculated implicitly using second order Adams-Moulton to yield an approximate solution for the velocity field.

In the current work, we used a non-uniform Cartesian mesh with 3.4 million cells with the dimensions of $300 \times 300 \times 250 \mathrm{~mm}$ in the $\mathrm{x}, \mathrm{y}$ and $\mathrm{z}$ directions respectively. Initial investigations for the grid sensitivity were carried out and more details can be found in [25] and [27]. The mean axial velocity distribution for the fuel inlet and mean axial and swirling velocity distributions for the air annulus are specified using the power law profiles such that:

$$
<U>=1.218 U\left(1-\frac{|y|}{\delta}\right)^{1 / 7}
$$

Here $U_{j}$ is bulk velocity, $y$ is the radial distance from the jet centre line and $\delta=1.01 R_{j}, R_{j}$ is fuel jet radius of $1.8 \mathrm{~mm}$. The factor 1.218 is chosen to ensure correct mass flow at the inlet. The fluctuations are generated from Gaussian random number generators which are then added to the mean velocity profiles such that the inflow has turbulent kinetic energy levels obtained from the experimental data. The method of turbulence generation using a Gaussian random number generator especially for complex swirl flow configurations is consistent and shows good agreement with other inflow generation methods for the Sydney swirling flames [27]. 
A top hat profile is used as the inflow condition for the mixture fraction. At the outflow plane, a convective outlet boundary condition is used for velocities and a zero normal gradient condition is used for the mixture fraction. A free slip boundary condition is applied at solid walls. Each simulation was carried out for $0.12 \mathrm{~s}$ and different sampling periods were studied to confirm that the solutions sufficiently converged.

\section{Results and discussion}

This section presents a detailed description of LES calculations for two different nonpremixed swirling flames known as SM1 and SM2 [20][21][22]. To validate the LES results, first we present the comparisons between LES calculations and experimental measurements. Then focus on the analysis of instability for flame SM1 followed by the analysis of instability for flame SM2 [22].

\subsection{Validation studies}

Before presenting the principle results of this study, the accuracy of the current LES results should be addressed. Here we consider the experimental data of flame SM1 [20] to validate our numerical results obtained from the LES calculations.

Fig. 2 shows the isosurface of the negative mean axial velocity at a value of $-0.1 \mathrm{~m} / \mathrm{s}$ for the flame SM1. The plot also shows the expansion of the upstream recirculation zone and downstream VB bubble. The intermediate region between the two recirculation zones is highly unstable in nature and appears to show a flapping behaviour. Here, LES appears to be very successful in reproducing all the flow features seen in the experiments. Stagnation region for the upstream recirculation zone where the mean axial velocity is zero is just above $40 \mathrm{~mm}$ which was observed 
in the experiments to be around $43 \mathrm{~mm}$. Fig. 3 shows the comparisons of the time averaged mean axial (left side) and mean swirling velocity (right side) at $\mathrm{x} / \mathrm{D}=0.136,0.8,1.4$ and 2.5 locations, where $\mathrm{D}=50 \mathrm{~mm}$ is the diameter of the bluff body and $\mathrm{R}=25 \mathrm{~mm}$ is the radius of the bluff body. The LES predictions captured a relatively short bluff body stabilised recirculation zone and also a second downstream central recirculation zone as observed by experimental measurements [20]. LES calculations further indicate that the axial and radial extents of the bluff body stabilised recirculation zone and swirl induced central recirculation zones are in close agreement with experimental observations. Despite a slight over prediction at $\mathrm{x} / \mathrm{D}=0.136$ and 1.4 , the mean swirling velocities also agree well with the experimental measurements. The over prediction can most likely be attributed to the shear layer instability and central jet precession. The overall agreement between experimental and simulated time averaged mean axial and swirl velocities are good at all considered axial locations. Shown in Fig.4 is the time averaged axial (left side) rms velocity and swirling (right side) rms velocity. The calculated rms axial velocity slightly underestimates at $\mathrm{x} / \mathrm{D}=1.4$ and rms swirl velocity overestimates at $\mathrm{x} / \mathrm{D}=0.8$ and 1.2. The central recirculation zone appears in this region and hence contains high turbulence on the boundary of the recirculation zone and thus small discrepancies are apparent. Despite the small discrepancies, the comparison between LES calculations and experimental measurements for the rms velocities are good at most axial locations.

Fig. 5 shows the time averaged mean mixture fraction (left side) and mean temperature (right side) at $\mathrm{x} / \mathrm{D}=0.2,0.8$ and 1.5 . The LES mean mixture fraction under predicts at $\mathrm{x} / \mathrm{D}=0.2$ and over predicts on the centreline at $\mathrm{x} / \mathrm{D}=1.5$. Since the mean 
temperature depends on the mean mixture fraction, the radial plots of the mean temperature also have the similar discrepancy at these axial locations. The overall agreement between computations and measurements for the time averaged mean mixture fraction and mean temperature are good and the calculations predict the trends which appeared in the temperature field. This validation study shows good agreement with experimental measurements which promotes confidence in presenting the main findings of this work. Here simulation results are analysed aiming to demonstrate the oscillations of two different swirling flames, SM1 and SM2 [22].

\subsection{Instability of flame SM1}

This section discusses the instability behaviour of the centre jet of flame SM1. The centre jet precession of flame SM1 was originally identified by Al-Abdeli et al. [22] in their experimental investigation of the Sydney swirl burner experimental data base managed by Masri’s group [20][21][22].

Fig. 6 (a-h) shows the LES predictions of central jet precession for flame SM1 at eight different time periods for the filtered axial velocities. The images indicate that the centre jet appears to move more into one side of the geometric centreline before crossing over to the other side. Hence the centre jet has an irregular random motion for flame SM1 which cannot be defined as regular time periodic behaviour. As seen in Fig.3, the present calculations observed the large scale wobbling motion of the jet tip. However, the experimental group observed a periodic (cyclic) variation of the centre jet for the other Sydney swirl flames and this instability precession is termed as Mode I [22]. Therefore, here we cannot define the centre jet precession as Mode I instability since it does not show cyclic variation with respect to time due to the conditions that have been adopted for this flame. According to Al-Abdeli et al. [22], the experimental 
measurements also observed a similar wobbling behaviour for the centre jet and for flame SM1 and confirms that the LES results are successful for detecting this irregular centre jet precession for this flame.

Furthermore in flame SM1, the experimental data found some peaks of the power spectrum for some frequency values in the flow field at downstream centreline axial stations. Therefore, we have generated the power spectrum using LES data to analyse the peak values which coincide with the experimental observations. Fig. 7 shows the power spectrum of the axial velocity for flame SM1. The plot has been produced for specifically selected centreline axial location, because it is perfectly located upstream of the spatial position where downstream recirculation occurs (Fig. 2). To analyse the unsteady oscillations of the flame SM1, monitoring points were selected from both sides of the centreline at $\mathrm{x}=60 \mathrm{~mm}$ downstream from the burner exist plane, which is similar to experimentally considered position. The power spectrum is constructed by applying the Fast Fourier Transform (FFT) for the instantaneous filtered axial velocity. The power spectrum indicates the presence of peaks at intermediate frequencies. Particularly, LES based power spectrum shows a distinct peak at approximately a frequency value of $75 \mathrm{~Hz}$ while experimental group observed peaks in the frequency range of approximately $63-68 \mathrm{~Hz}$ [22]. This is an interesting finding compared to the experimental observation as the occurrence of downstream recirculation zone further strengthens the mixing of an already turbulent jet. In addition, the considered location is situated near the top of the bluff body stabilized recirculation zone, which may also cause some vortex shedding. Hence, the situation is much more complex than the upstream central jet irregular precession as discussed in above paragraph. Since both modelling and experimental data have identified the 
peaks of the power spectrum for this frequency range, one can imagine an existence of another instability mode in the intermediate region between a bluff body stabilised first recirculation zone and a swirl stabilised second recirculation zone for this particular flame (SM1). The possibility of defining another Mode of instability has been ruled out at this moment and further investigations from both the modelling and experimental evidences are required in order to make this claim.

\subsection{Instability of flame SM2}

Figs. 8 and 9 show snapshots of the filtered temperature and vorticity field of flame SM2. The temperature field exhibits pockets of high temperature in both the upstream recirculation zone and the downstream VB region. This flame is much more compact than SM1 [26], mainly due to the higher jet velocity and increased turbulence intensity. As shown in Fig. 9, the large vortical structures arise in the shear layers at downstream. These swirl induced vorticies eventually break up into small-scale eddies and finally dissipate due to turbulent diffusion and viscous damping.

This section analyses the time varying behaviour of flame SM2, which is the other flame of the SM flame series experimentally investigated by Al-Abdeli et al. [22] and we discuss the instability associated with bluff body stabilised recirculation zone. AlAbdeli et al. [22] originally examined the instability associated with the bluff body stabilised recirculation zone and identified expansion and collapse of the recirculation zone known as "puffing” which they defined as Mode II instability. Here we attempt to identify the Mode II instability using the LES data and discuss the similarities with already established experimental observations [22]. For this, we study the time periodicity of the recirculation zone using various snapshots and power spectrum. Fig. 
10 (a-f) shows the LES predictions of sequential collapse and then expansion of the bluff body stabilised recirculation zone at six different time periods for the flame SM2. The snapshots show the contour plot of filtered axial velocity for the bluff body stabilised recirculation zone where solid line indicates the boundary of the recirculation zone and dashed lines indicate the negative filtered axial velocity. The considered range for the contour values ( $0 \mathrm{~m} / \mathrm{s}$ to $-14 \mathrm{~m} / \mathrm{s}$ ) are shown in Fig. 10 (a). Fig.10 (a) shows a snapshot at one particular time and the recirculation zone is clearly seen to reduce more in Fig. 10 (b) and (c) for the next two time intervals. The recirculation zone again starts to expand in Fig. 10 (d) and (e) and eventually forms a similar shape as the initial snapshot in Fig. 10 (a) (Fig. 10 (f)). Hence the time dependent snapshots show a sequential collapse/contraction and then expansion of the recirculation zone similar to that found in the experimental observation referred as “puffing” motion and defined as Mode II instability [22]. It is worth while to note that the Mode II instability has only occurred in Sydney burner reacting swirling flames and it has not been identified in the Sydney swirl burner isothermal swirling jets either numerically [28] or experimentally [22]. Thus we can conclude that the addition of combustion creates this instability mode due to the combination of flow conditions and combustion heat release.

In addition to the snapshot analysis as noted above, in the current work we also study the power spectrum generated from LES data for selected positions inside the recirculation zone. To analyse the time periodicity in the recirculation zone, a pair of monitoring points around the envelope of the bluff body stabilised recirculation zone are considered [22]. The power spectrum is constructed by applying the Fast Fourier Transform (FFT) for the instantaneous filtered axial velocity. Fig. 11 shows the power 
spectrum associated with SM2 at the envelope of the bluff body stabilised recirculation zone. The power spectrum of flame SM2 shows a peak at low frequency which becomes more distinct around $\sim 20 \mathrm{~Hz}$. The peak around $\sim 20 \mathrm{~Hz}$ is an attribute to Mode II instability and the identification of this peak further demonstrates the usefulness of the LES technique for simulation of complex unsteady flames and combustion dynamics.

\section{Conclusions}

Modelling of instabilities has been conducted using LES for the laboratory scale turbulent unconfined swirling flames experimentally investigated at Sydney University [20-22]. The simulations captured the centre jet precession for flame SM1 and the recirculation zone precession for flame SM2. The unsteady data from the LES calculations along with a Fast Fourier Transform (FFT) algorithm have been used for data analysis. Various snapshots and power spectra indicate the irregular precession of centre jet for flame SM1 and precession behaviour further downstream in the region between the upstream and downstream recirculation zones. Results show that the instability arises in a bluff body stabilised recirculation zone for flame SM2. This has been referred as Mode II instability and has not been identified in Sydney burner isothermal jets in either experimental or numerical investigations [21][28]. The current study, along with previous work [27-28] demonstrated that the LES technique can be used for swirl combustion applications, especially to capture the unsteady flame properties and instability behaviour. We intend to extend this work to explore the underlying mechanisms responsible for driving unsteady motions in gas turbine combustion systems. 


\section{References}

[1] Lieuwen, T, Yang, V. Combustion Instabilities in gas turbine engines: operational experience, fundamental mechanisms, and modelling. Progress in Astronautics and Aeronautics, AIAA publisher 2005

[2] Syred, N. A review of oscillation mechanisms and the role of the precessing vortex core (PVC) in swirl combustion systems. Prog. Energy. Combust. Sci. 2006; 32:93-161

[3] Froud, D, O’Doherty, T, Syred, N. Phase averaging of the precessing vortex core in a swirl burner under piloted and premixed combustion conditions. Combust. Flame $1995 ; 100: 407-417$

[4] Syred, N, Fick, W, O’Doherty, T, Griffiths, AJ. The effect of the precessing vortex core on combustion in swirl burner. Combust. Sci. Tech. 1997 125, pp. 139-157

[5] Sankaran, V, Menon, S. LES of spray combustion in swirling flows, J Turbulence 2002; 3:11-23

[6] Apte, SV, Mahesh, K, Moin, P, Oefelein, JC. Large eddy simulation of swirling particle laden flows in a coaxial jet combustor, Int J Multiphase Flows 2003; 29(8): $1311-1331$

[7] Oefelein, JC, Sankaran, V, Drozda, TG. Large eddy simulation of swirling particle laden flow in a model axisymmetric combustor, Proc. Combust. Inst. 2007; 31 (2): 2291-2299

[8] Mongia, HC. Recent progress in comprehensive modelling of gas turbine combustion, AIAA 2008; 1445

[9] Pitsch, H. Large eddy simulation of turbulent combustion, Ann. Rev. Fluid Mech. 2006; 38:453-482 
[10] Kempf, A. LES validation from experiments, Flow Turb. 2008 Combust. Online first

[11] Lu, X, Wang, S, Sung, H, Hsieh, S, Yang, Y. Large eddy simulations of turbulent swirling flows injected into a dump chamber, J Fluid Mech. 2005; 527:171-195

[12] Wang, S, Yang, V, Hsiao, G, Hsieh, S, Mongia, HC. Large eddy simulations of gas turbine swirl injector flow dynamics, J Fluid Mech. 2007; 583:99-122

[13] Oefelein, JC. Large eddy simulation of turbulent combustion processes in propulsion and power systems, Prog Aero Sciences 2006; 42: 2-37

[14] Grinstein, FF, Liu, NS, Oefelein, JC. Combustion Modelling and Large eddy simulation: Development and validation needs for gas turbines, AIAA J. Special section 2006; 44: 673-741

[15] Patel, N, Menon, S. Simulation of spray-turbulence-chemistry interactions in a lean direct injection combustor, Combust. Flame 2008; 153: 228-257

[16] Roux, A, Gicquel, LYM, Sommerer, Y, Poinsot, TJ. Large eddy simulation of mean and oscillating flow in a side dump ramjet combustor, Combust Flame 2008; 152: $154-176$

[17] Pierce, CD, Moin, P. Progress variable approach for LES of non-premixed turbulent combustion, J Fluid Mech. 2004; 504: 73-97

[18] Reveillon, J, Vervisch, L. Spray vaporization in nonpremixed turbulent combustion modelling: a single droplet model, Combust Flame 2000; 121: 75-90

[19] Angelberger, C, Veynante, D, Egolfopoulos, F, Poinsot, T. Large eddy simulations of combustion instabilties in premixed flames, Proc Summer Program Center for Turbulence Research, Stanford University, CA, 1998; 61-83 [20] Al-Abdeli, YM, Masri, AR. Stability Characteristics and flow fields of turbulent non-premixed swirling flames, Combust. Theo. Mode. 2003; 7: 731-766 
[21] Al-Abdeli, YM, Masri, AR. Precession and recirculation in turbulent swirling isothermal jets, Combst. Sci. Tech. 2004; 176: 645-665

[22] Al-Abdeli, YM, Masri, AR, Marquez, GR, Staner, S. Time-varying behaviour of turbulent swirling nonpremixed flames, Combust. Flame 2006; 146: 200-214

[23] El-Asrag, H, Menon, S. Large eddy simulation of bluff-body stabilised swirling non-premixed flame, Proc. Combust. Inst. 2007; 31: 1747-1754

[24] James, S, Zhu, J, Anand, MS. Large eddy simulation of turbulent flames using filtered density function method, Proc. Combust. Inst. 2007; 31: 1737-1745

[25] Malalasekera, W, Ranga Dinesh, KKJ, Ibrahim, SS, Kirkpatrick, MP. Large eddy simulation of isothermal turbulent swirling jets. Combust. Sci. Tech. 2007; 179: 14811525

[26] Malalsekera, W, Ranga Dinesh, KKJ, Ibrahim, SS, Masri, AR. LES of recirculation and vortex breakdown in swirling flames. Combust. Sci. Tech. 2008; 180: 809-832

[27] Kempf, A, Malalasekera, W, Ranga Dinesh, KKJ, Stein, O. Large eddy simulation with swirling non-premixed flames with flamelet model: A comparison of numerical methods. Flow Turb. Combust. 2008; 81: 523-561

[28] Ranga Dinesh, KKJ, Kirkpatrick, MP. Study of jet precession recirculation and vortex breakdown in turbulent swirling jets using LES, Int. J. Comput. and Fluids 2009; 38: 1232-1242

[29] Smagorinsky, J. General circulation experiments with the primitive equations. M. Weather Review. 1963; 91: 99-164

[30] Piomelli, U, Liu, J. Large eddy simulation of channel flows using a localized dynamic model. Phy. Fluids 1995; 7: 839-848 
[31] Pitsch, H. A C++ computer program for 0-D and 1-D laminar flame calculations, RWTH, Aachen, 1998

[32] Bowman, CT, Hanson, RK, Davidson, DF, Gardiner, WC, Lissianki, V, Smith, GP, Golden, DM, Frenklach, M, Goldenberg, M. GRI 2.11, 2006

[33] Branley, N, Jones,WP. Large eddy simulation of a turbulent non-premixed flame, Combust. Flame 2001; 27: 1914-1934

[34] Kirkpatrick, MP. Large eddy simulation code for industrial and environmental flows. PhD Thesis, University of Sydney, Australia, 2002

[35] Ranga Dinesh,, KKJ. Large eddy simulation of turbulent swirling flames. PhD Thesis, Loughborough University, UK, 2007

[36] Leonard, BP. SHARP simulation of discontinuities in highly convective steady flows, NASA Tech. Memo 1987; 100240 


\section{Figure Captions}

Fig. 1. Schematic drawing of the Sydney swirl burner

Fig. 2. Iso-surface of upstream recirculation zone and downstream VB bubble, here the units of $\mathrm{x}, \mathrm{y}$ and $\mathrm{z}$ are in metre

Fig. 3. Comparison of mean axial velocity (left side) and mean swirling velocity (right side). Lines represent LES results, and symbols represent experimental measurements Fig. 4. Comparison of rms axial velocity (left side) and rms swirling velocity (right side). Lines represent LES results, and symbols represent experimental measurements. Fig. 5. Comparison of mean mixture fraction (left side) and mean temperature (right side). Lines represent LES results, and symbols represent experimental measurements Fig. 6. Irregular jet precession of flame SM1 visualised by filtered axial velocity Fig. 7. Power spectrum of flame SM1 at $60 \mathrm{~mm}$ downstream from the burner exit plane

Fig. 8. Snapshot of flame temperature for flame SM2

Fig. 9. Snapshot of vorticity field for flame SM2

Fig. 10. Mode II instability in flame SM2 identified using LES visualised by filtered axial velocity

Fig. 11. Power spectrum of flame SM2 at the envelope of the recirculation zone 
Figures

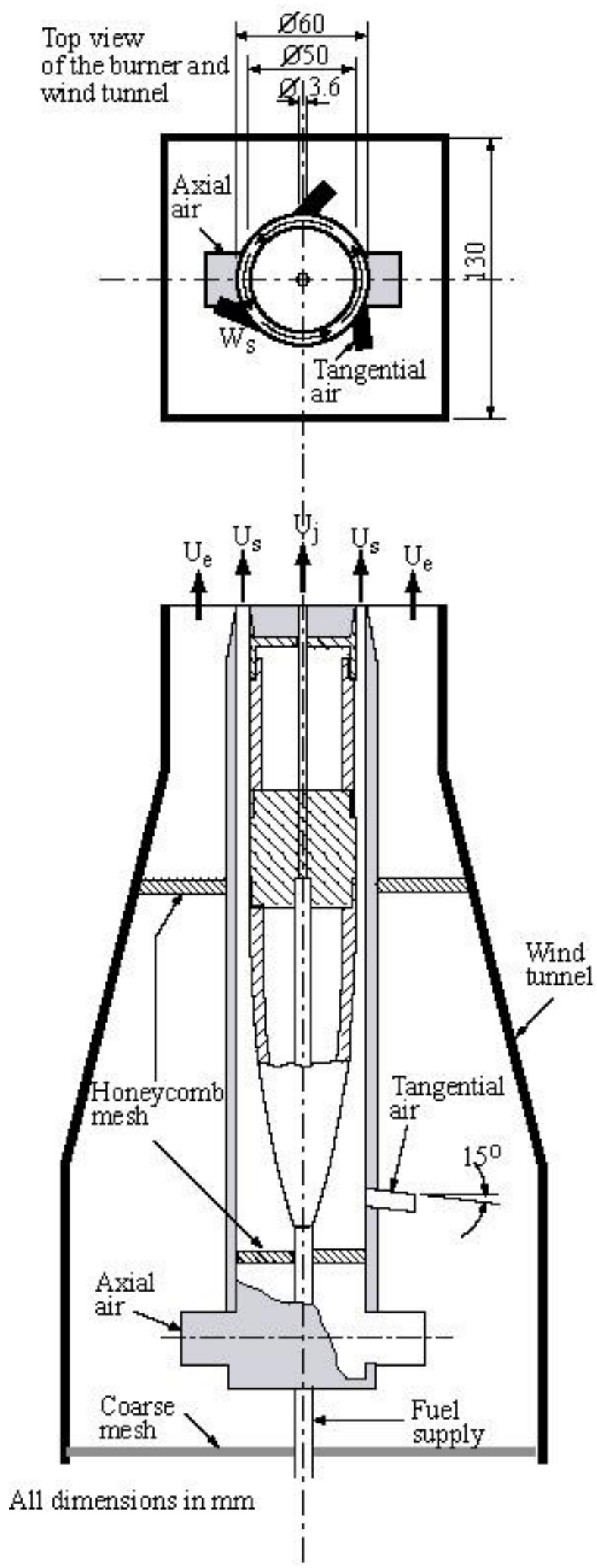

Fig. 1. Schematic drawing of the Sydney swirl burner 


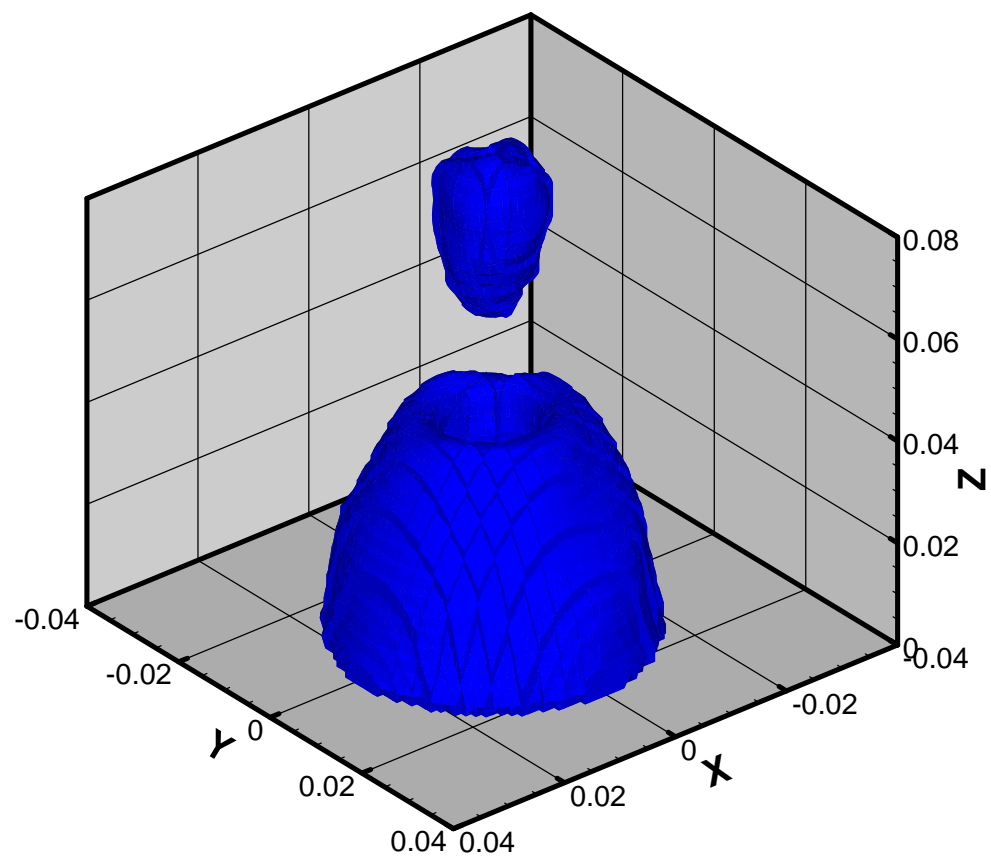

Fig. 2. Iso-surface of upstream recirculation zone and downstream VB bubble (-0.1 $\mathrm{m} / \mathrm{s}$ ), here the units of $\mathrm{x}, \mathrm{y}$ and $\mathrm{z}$ are in metre 

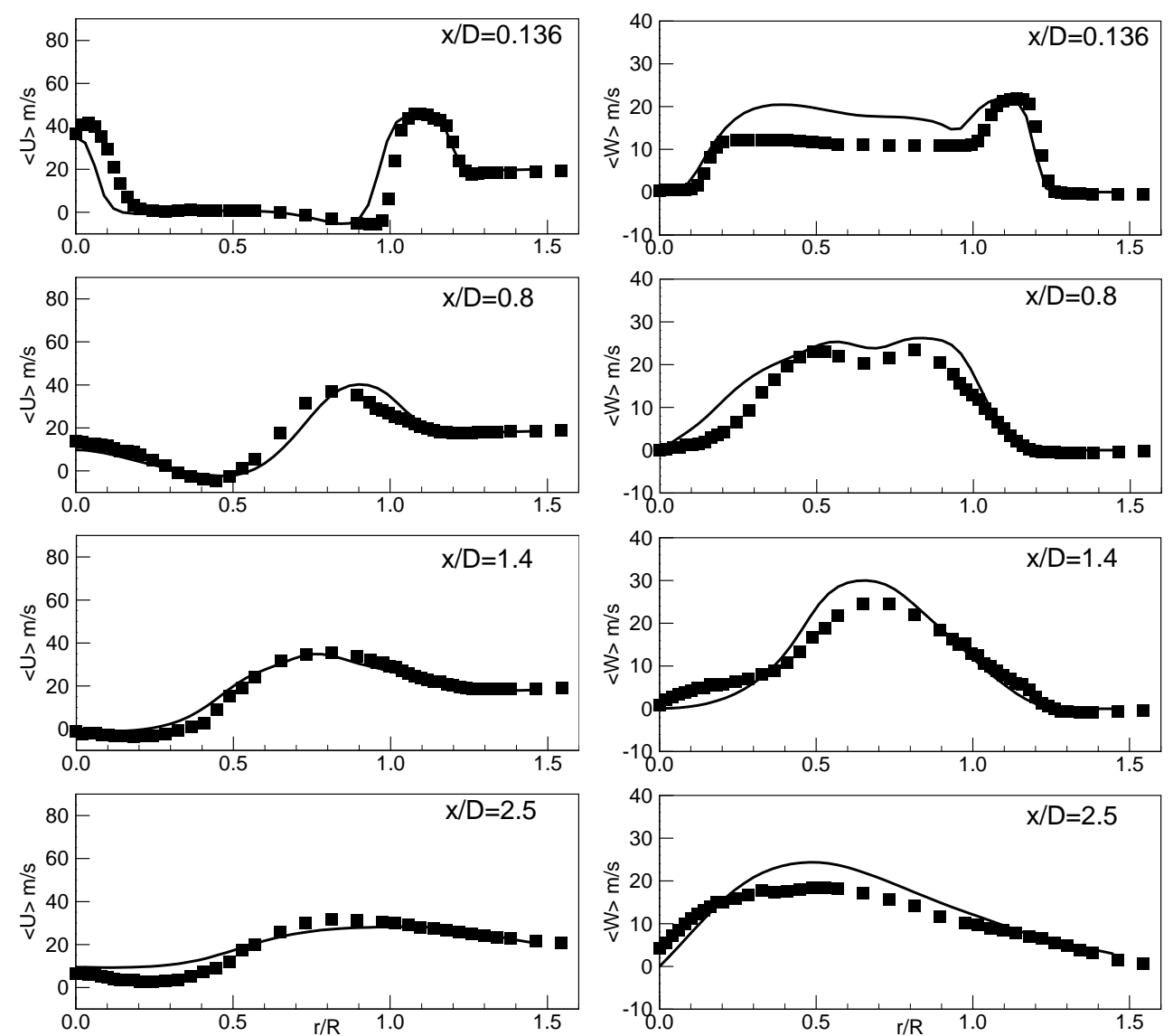

Fig. 3. Comparison of mean axial velocity (left side) and mean swirling velocity (right side). Lines represent LES results, and symbols represent experimental measurements. 

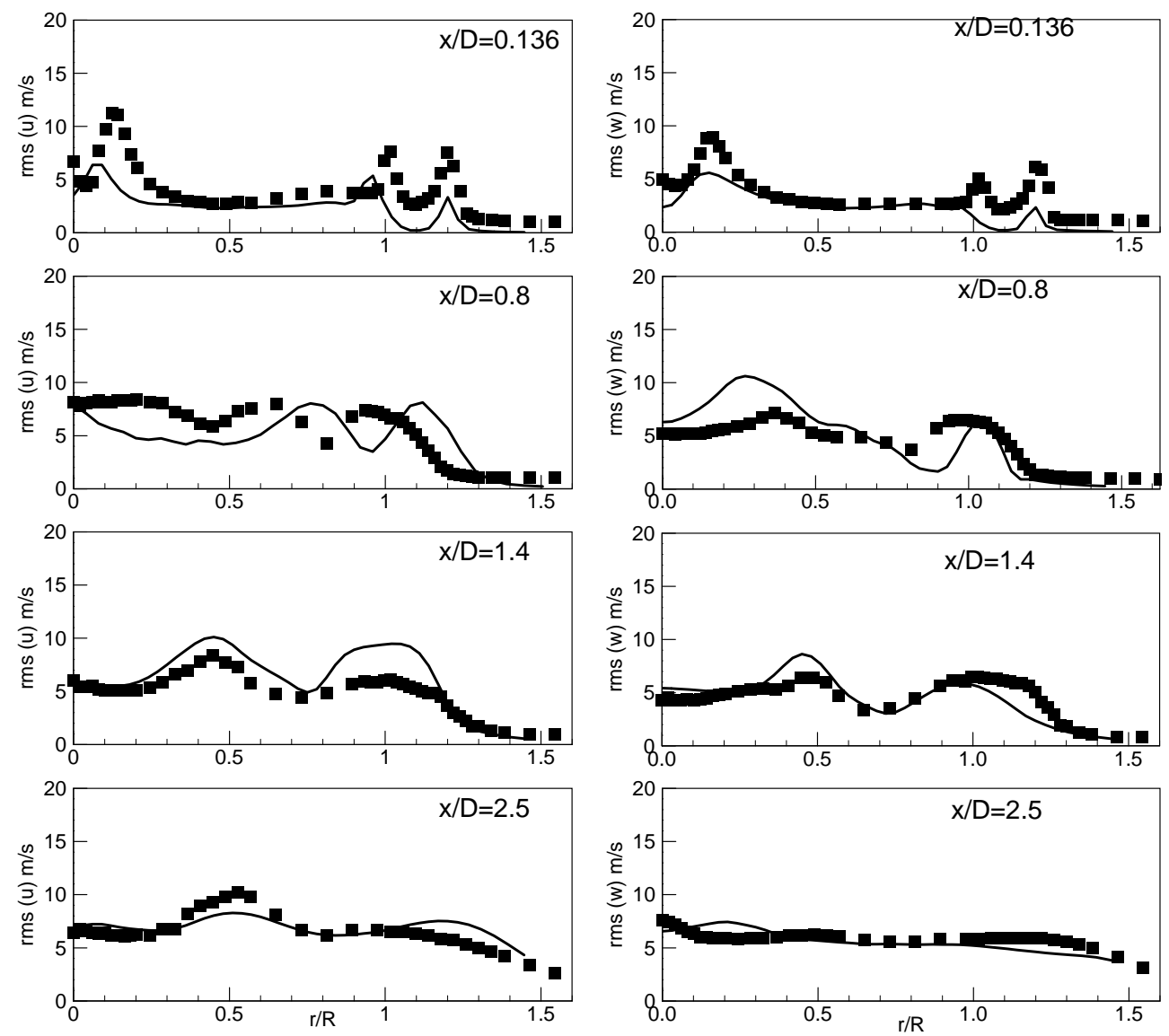

Fig. 4. Comparison of rms axial velocity (left side) and rms swirling velocity (right side). Lines represent LES results, and symbols represent experimental measurements. 

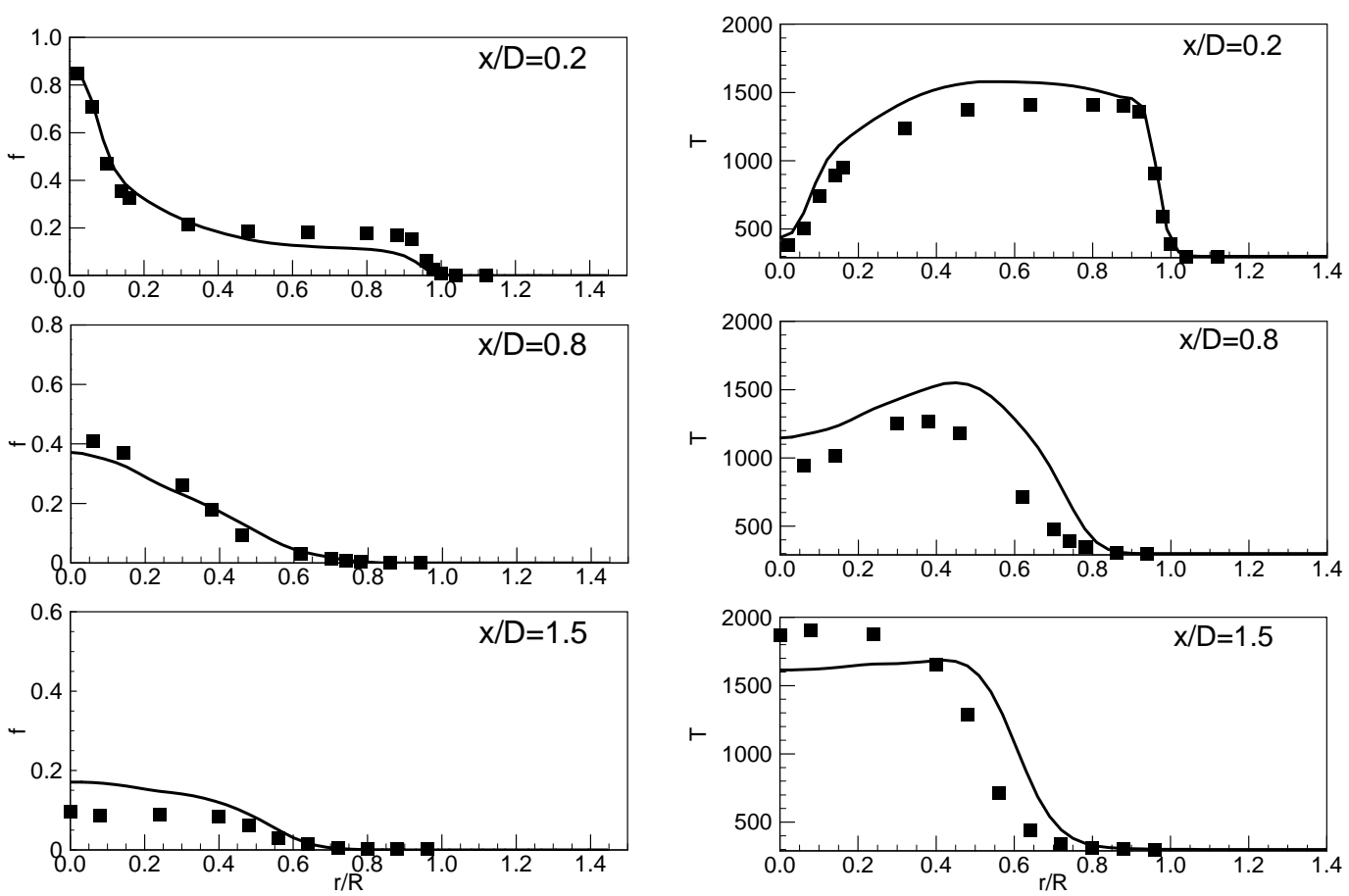

Fig. 5. Comparison of mean mixture fraction (left side) and mean temperature (right side). Lines represent LES results, and symbols represent experimental measurements. 

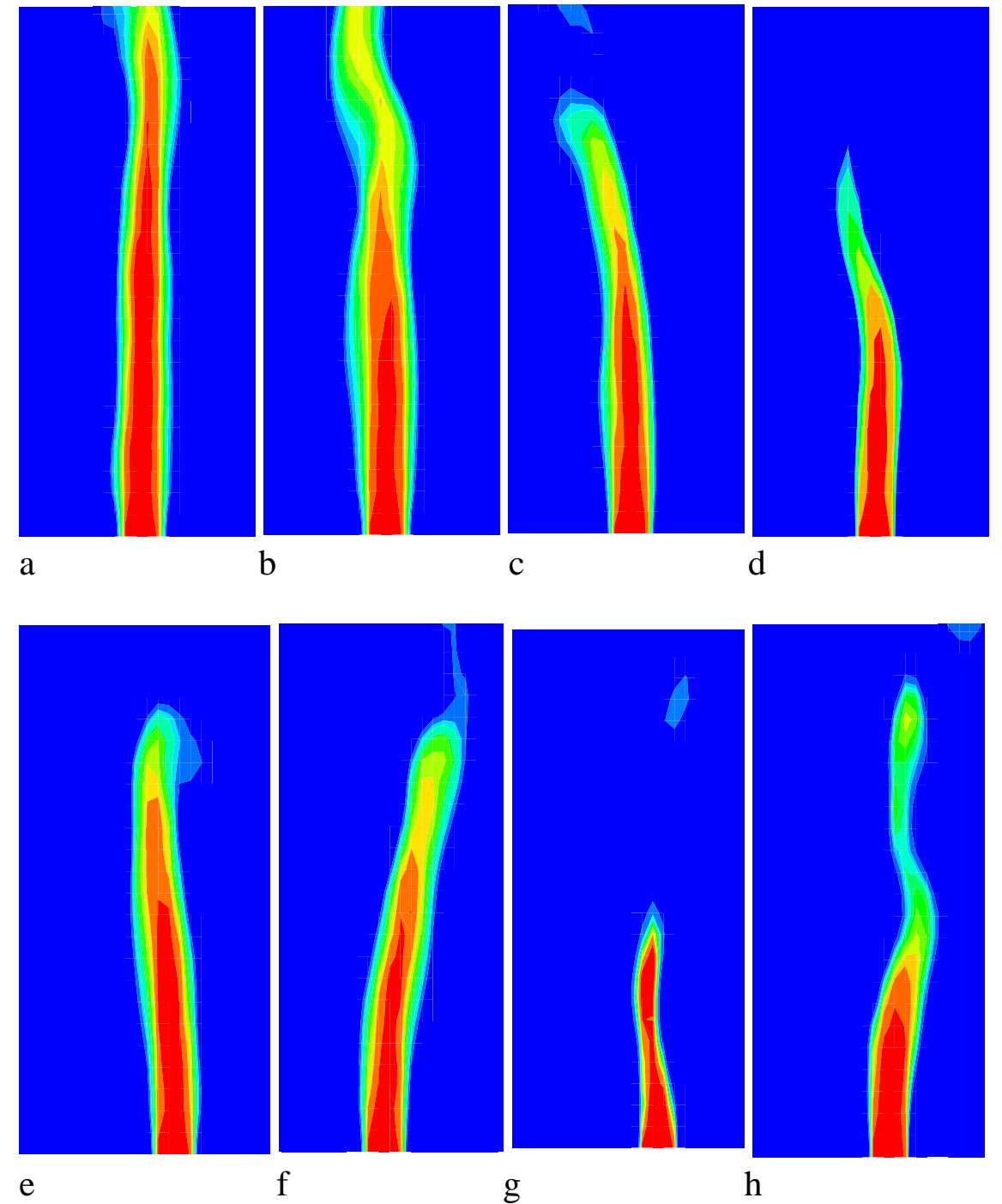

Fig. 6. Irregular jet precession of flame SM1 visualised by filtered axial velocity at eight different time periods (a-h) 


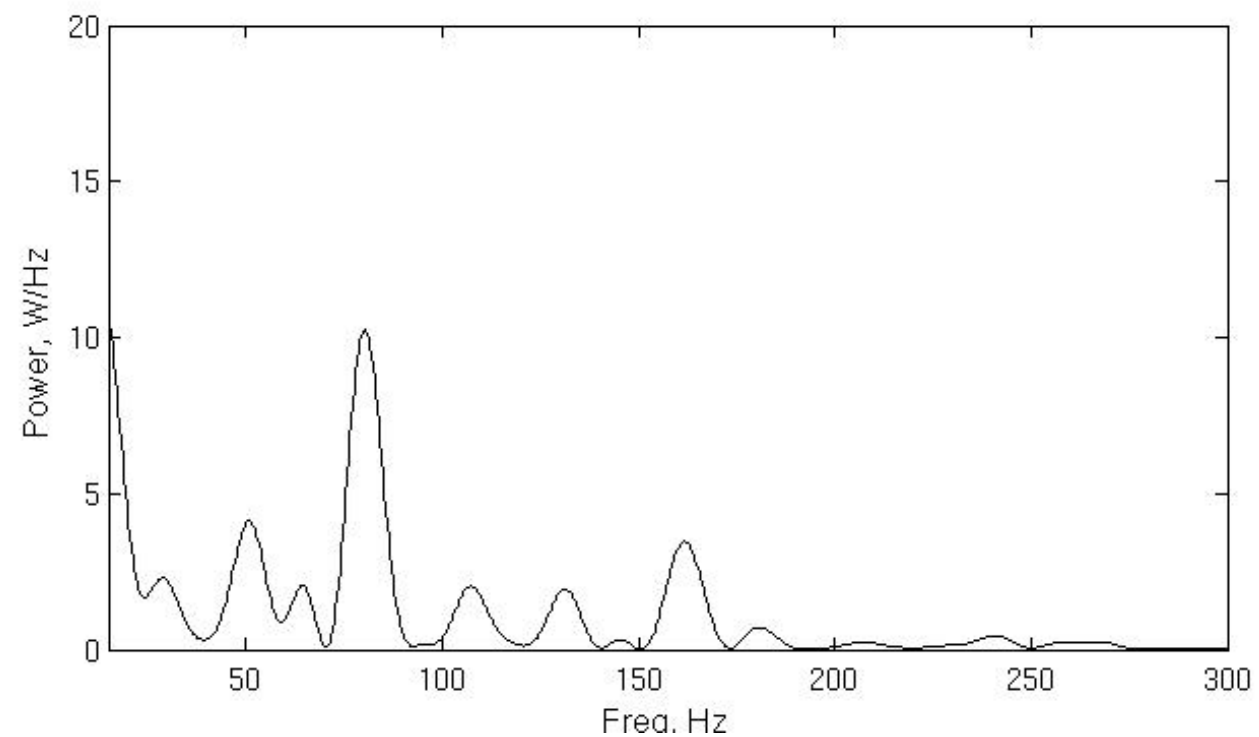

Fig. 7. Power spectrum of flame SM1 at $60 \mathrm{~mm}$ downstream from the burner exist plane 


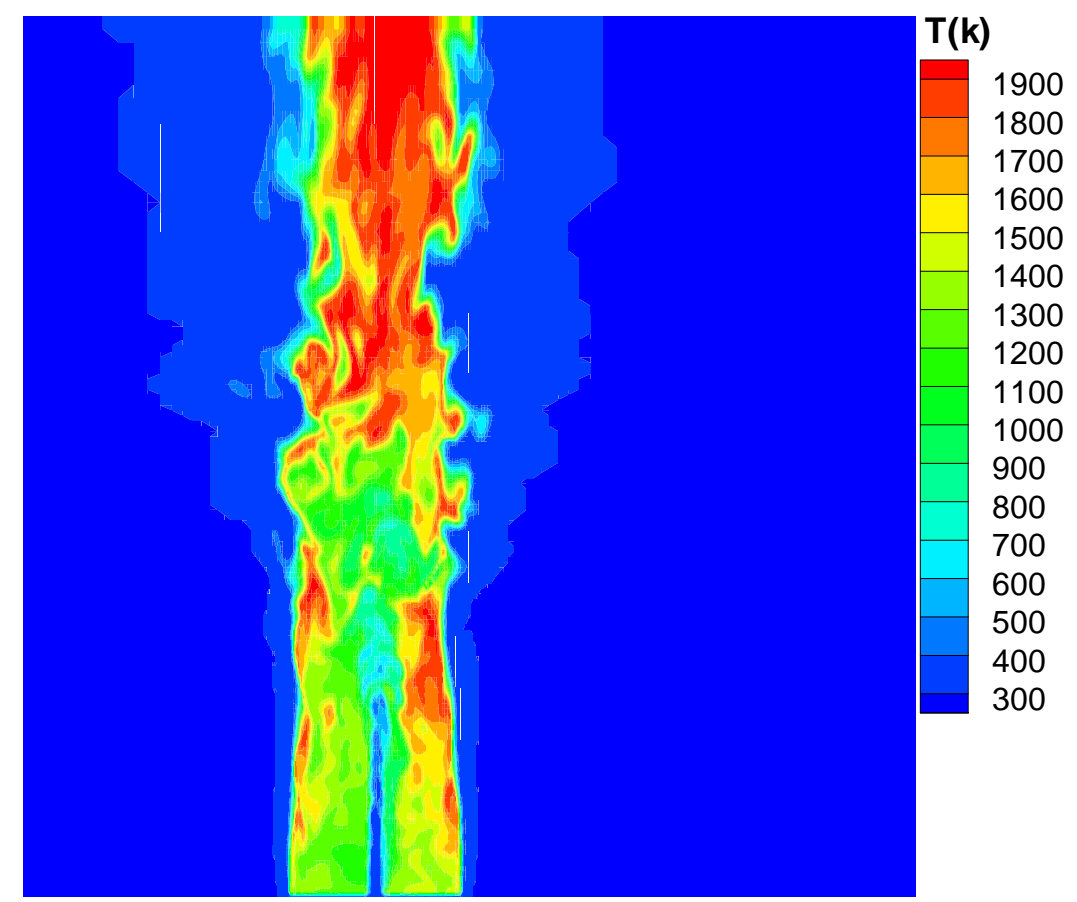

Fig. 8. Snapshot of flame temperature for flame SM2

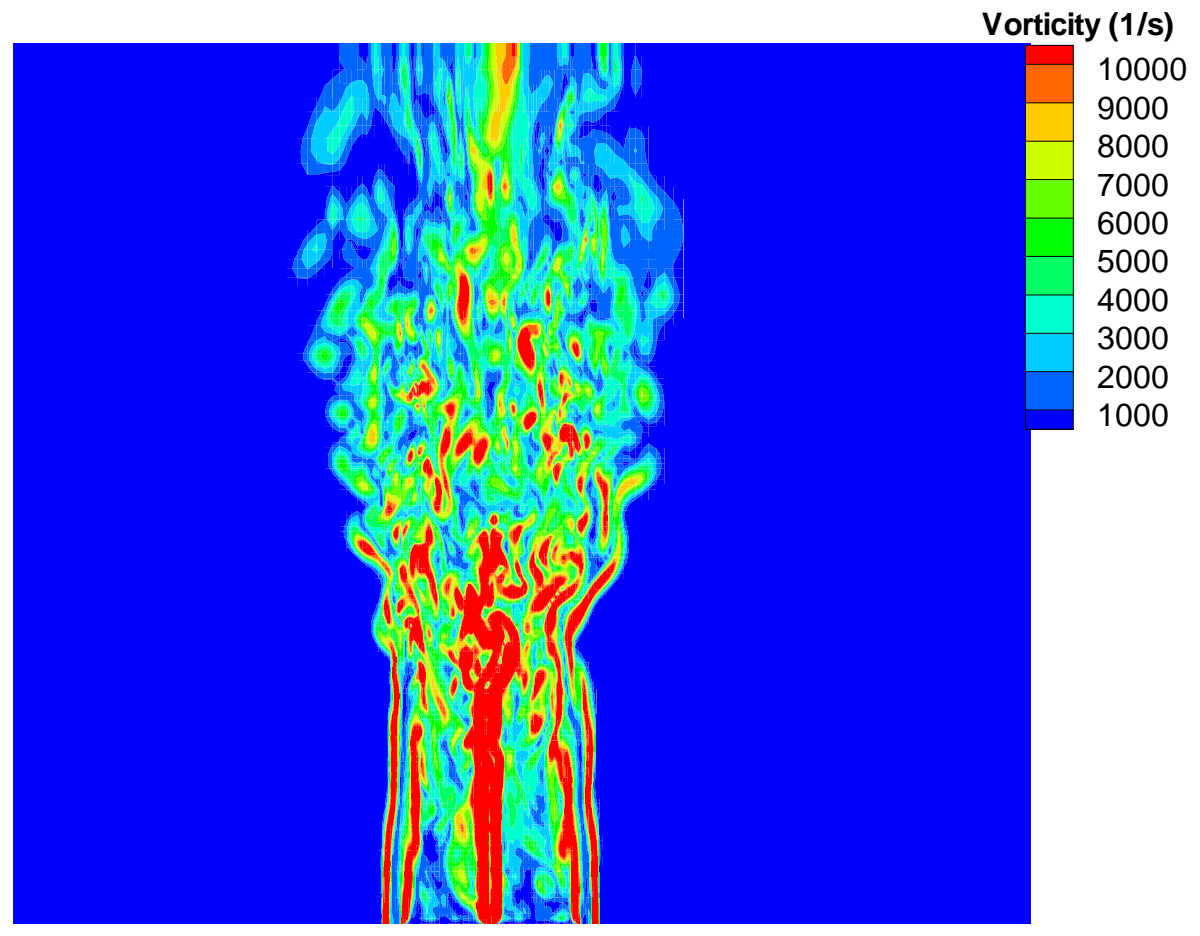

Fig. 9. Snapshot of vorticity field for flame SM2 


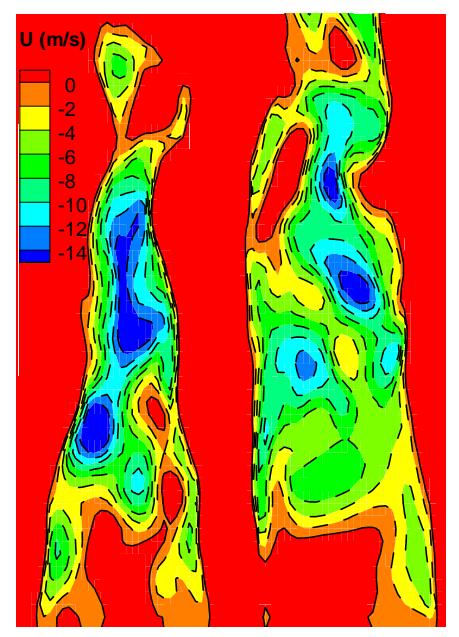

a

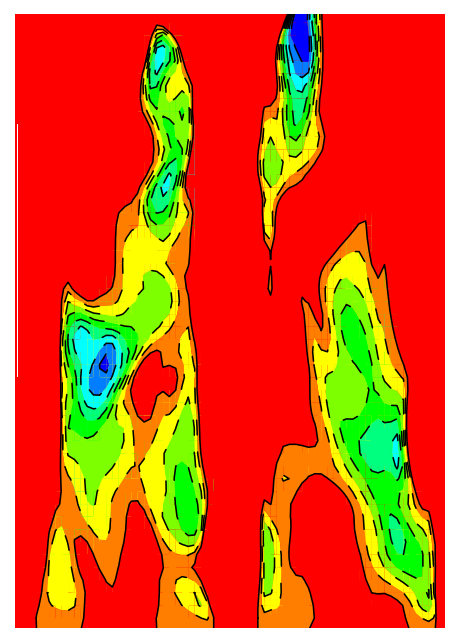

d

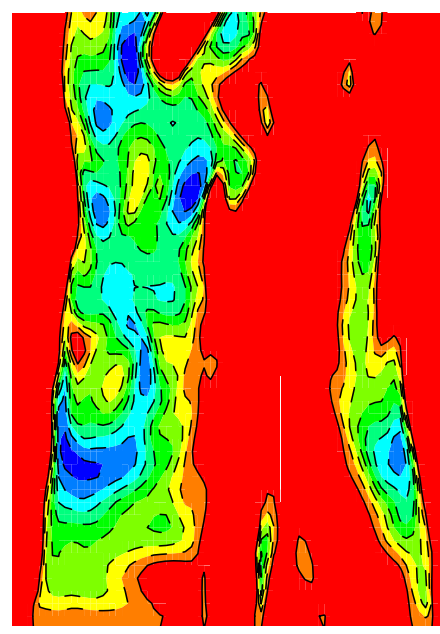

b

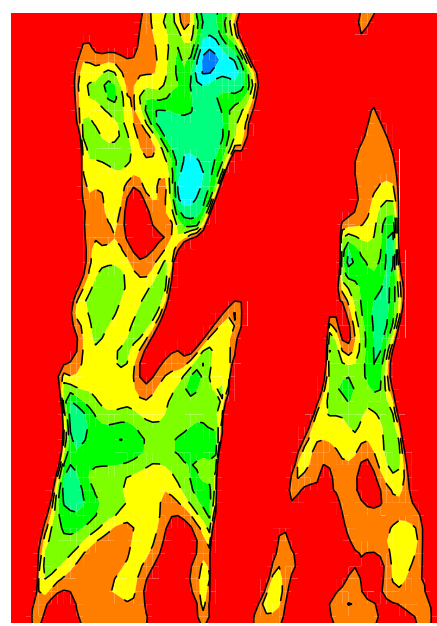

e

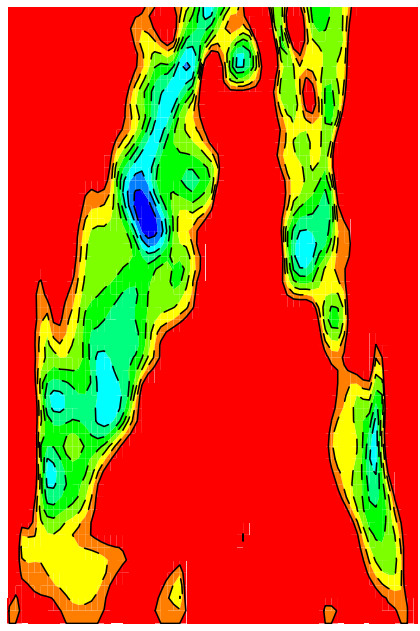

C

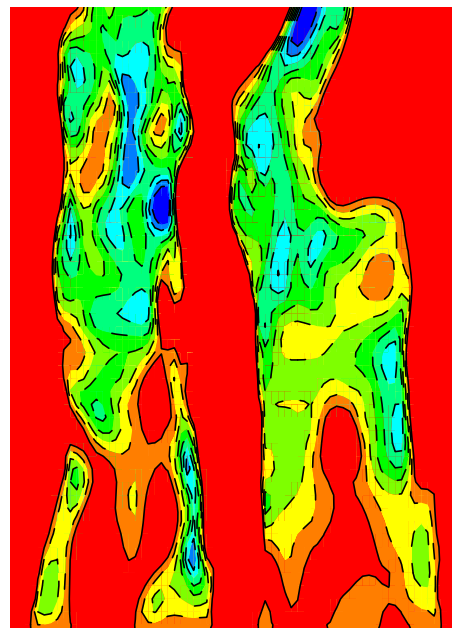

f

Fig. 10. Mode II instability in flame SM2 identified using LES visualised by filtered axial velocity at six different time periods (a-f) 


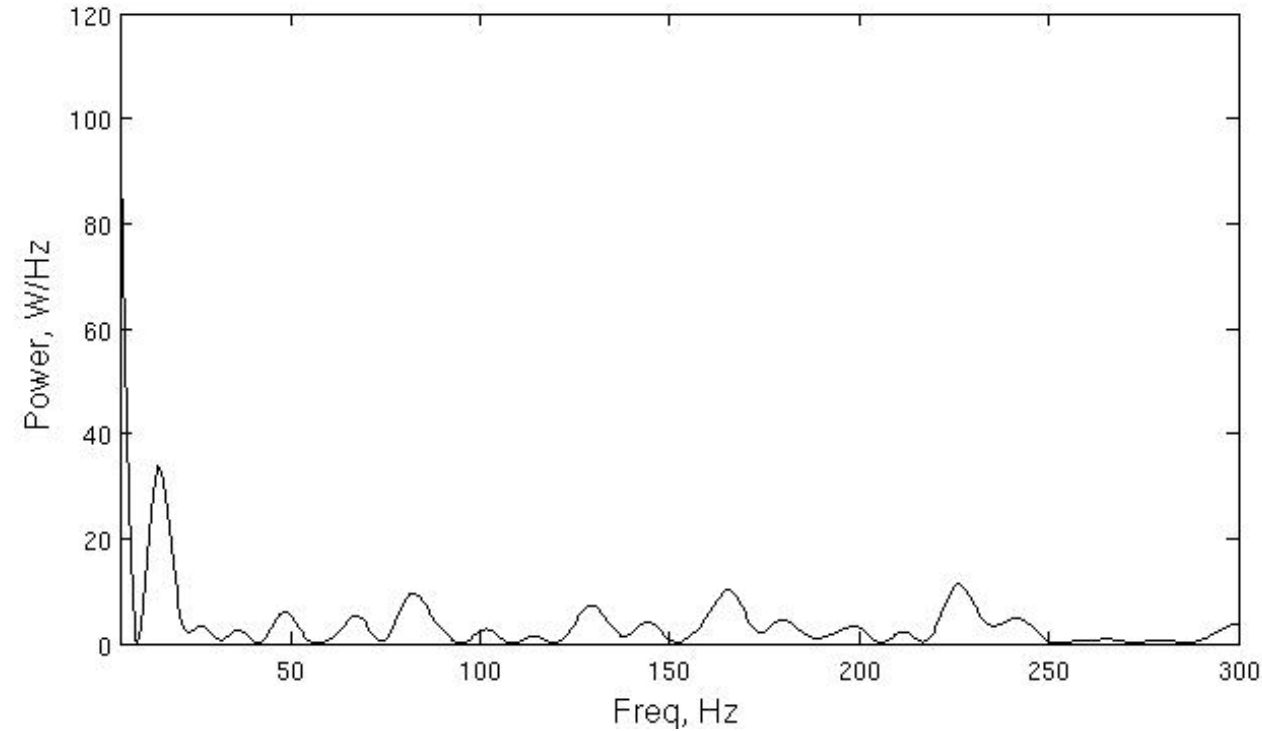

Fig. 11. Power spectrum of flame SM2 at the envelope of the recirculation zone 